\title{
Coverage Holes Compensation Algorithms Based on Event-Driven Strategy in Wireless Sensor Networks
}

\author{
Zeyu Sun ${ }^{12}$, Yali Yun ${ }^{1}$, Yalin $\mathrm{Nie}^{1}$ and Yuanbo $\mathrm{Li}^{1}$ \\ ${ }^{1}$ School of Computer and Information Engineering, Luoyang Institute of Science \\ Technology, Henan, Luoyang 471023, China; \\ ${ }^{2}$ School of Electronics and Information Engineering, Xi' an Jiaotong University, \\ $X i$ 'an, Shanxi 710049 China \\ E-mail:lylgszy@163.com
}

\begin{abstract}
The process of random deployments, Coverage holes' phenomena were appeared in wireless sensor network system. This paper presents a probabilistic model by means of event-driven policy coverage holes' compensation method (Coverage Holes Compensation Algorithms Based on Event-Driven Strategy, CHCAEDS). Firstly, the characteristics of random deployment verified, given the random deployment of representation, followed by the use of probabilistic knowledge within the surveillance area coverage desired and the number of nodes is solved using the minimum number of nodes in order to achieve maximum coverage area; and finally, simulation experiment show, CHCAEDS algorithm with other algorithms in the network life cycle and the algorithm running time increased by $12.59 \%$ and $10.82 \%$.
\end{abstract}

Keywords: wireless sensor networks; coverage quality; coverage holes; event-driven strategy

\section{Introduction}

With the rapid advances of information technology, wireless sensor network (WSN) that get an unprecedented rapid development, has been widely used in military defense, health care, transportation, environmental monitoring, disaster relief, smart home and other engineering fields [1-2]. Wireless sensor network is a new type of network architecture, which is formed by thousands of sensor nodes through self-organization. The main characteristic of behavior is that each node has the abilities of sensing, computing, communicating and storage [3]. Wireless sensor network is a network architecture which organically integrated physical world and information world [4]. Coverage is a key problem in the wireless sensor networks, and it is also a core problem. Coverage directly determines coverage quality, network lifetime, the number of redundant nodes, and network quality of service in wireless sensor networks.

In recent years, experts and scholars from home and abroad have done a lot of research work on the coverage of wireless sensor networks. The radio relationship between the coverage quality of network, network connectivity and various parameters of the network system is given by the connected Coverage Protocol [5-6]; Literature [7] introduced the artificial intelligence algorithm of colony algorithm and particle swarm algorithm to complete the coverage of the entire monitoring network, in the coverage optimization stage, two kinds of intelligent algorithms are completed to optimize the global coverage, and finally, it achieves the completely covered of the monitoring area; a new algorithm of hole repairing is proposed, which is based on voronoi effective coverage area. Under meet the certain coverage quality [8], in view of the coverage holes, the algorithm can 
reasonably increase the working nodes in order to improve the current coverage, and find reasonable repair location information to ensure the connectivity of the whole network.

Due to the constraints and limitations of the wireless sensor network [9-10], such as: node energy is limited. Therefore, the following problems are mainly solved in the process of node coverage. First, it does not require completely coverage of the entire monitoring area, but the concerned target nodes are effectively covered; Second, when the sensor nodes are deployed, the random deployment is generally adopted, and how to avoid the phenomenon of coverage holes in the monitoring area; Third, how to reasonably deploy nodes and restrain the rapid consumption of node energy, at the same time, the minimum number of sensor nodes is used to achieve the effective coverage of the monitoring area, and in order to achieve the purpose of extending the network lifetime.

\section{Related Works}

In recent years, many scholars from home and abroad have carried out fruitful research about the coverage of wireless sensor networks, and have made some progress. A Scheduling Control Algorithm (SCA) is proposed in [11]. By solving the relationship function of the network connectivity and coverage quality, the probability that any target node in the monitoring area is covered by the sensor node is given, and it can dynamically adjust the position distribution of active nodes through the SCA algorithm, so that it can achieve the whole network energy balance. A distributed deployment node coverage algorithm is proposed in [12]. The monitoring area is divided into several different regions, the algorithm constitutes a Voronoi diagram and the geometric is calculated for each sub region. The affiliation between edges and angles of the maximum and minimum is given in the sub region, and the results are optimized by iteration. Finally, it can complete the coverage purpose for each sub region. The artificial intelligence algorithm of colony algorithm and particle swarm algorithm are used to solve the network coverage [13]. The idea is that local solution set can iterative refinement by two kinds of intelligent algorithm, at the same time, limit the local optimum "overflow"; In the aspect of global optimization, the local optimal solution set is optimized by the adaptive function, and the global optimal solution is achieved. Finally, the coverage of the whole network system is completed. A kind of optimization strategy for coverage holes is proposed in the [14]. The idea is that it can increase the sensor node reasonably and optimize the node in the condition of satisfying certain coverage, the method is based on the theoretical knowledge of geometric graphics to calculate the holes' area and the sensor nodes, and find the best position of holes' repair. Meanwhile, it can reduce the number of sensor nodes and complete the network connectivity. $k$-degree barrier coverage algorithm which is based on network lifetime maximization is proposed in the [15]. The algorithm provides coverage has the upper and lower solution method of theoretical significance; in the energy conversion of sensor nodes, the communication path between the sensor nodes are optimized by using a greedy algorithm, and ultimately achieve balance the network energy. An optimal coverage algorithm which is based on Event-Probability-Driven Mechanism (EPDM) is proposed in the [16]. Firstly, the network probability model is established, and the coverage rate of any sensor nodes in the monitoring area is solved; Secondly, the proof process of the $k$-degree coverage of the target node is given; finally, in the path selection, the ant colony algorithm is optimized to find the optimal path, and achieve the purpose of saving the network energy. Literature [17] proposes an EnergyEfficient Target Coverage Algorithm (ETCA). The algorithm is used to classify the target nodes by linear programming method, and the idea of clustering is used to effectively cover the set of multi-target. By calculating the residual energy of sensor nodes to achieve a balance, and achieve effective coverage of the monitoring area.

Based on the literature [16-17] algorithm, this paper establishes a model of the relationship between the sensor node and the multi-object node; On the basis of this, 
Coverage Holes Compensation Algorithms Based on Event-Driven Strategy (CHCAEDS) is proposed. The protocol is used to calculate the coverage expected value of the relationship between the sensor nodes and the multiple target nodes to ensure a reasonable coverage quality; then, the energy conversion of the whole network system is completed by the scheduling mechanism of sensor nodes, which can achieve the equilibrium of network energy.

\section{Quality of Coverage}

In order to further study, the coverage problem of wireless sensor networks [18-20], and make the problem simple and easy to operate. In this paper, the CHCAEDS algorithm is based on the following four assumptions:

(1) All sensor nodes' sensing radius and communication radius are disc-shaped at the initial time, and the energy of each node is the same.

(2) The sensor nodes are randomly deployed in monitoring area by a square, and ensure the node's sensing radius is far less than the length, boundary effect can be ignored.

(3) Each node has a unique identification code, and it does not depend on a certain location algorithm.

(4) The sensor nodes are isomerism form, and the synchronous clock [21].

Definition 1(Effective Coverage): When the target node is continuously covered by a finite number of sensor nodes, it is called effective coverage; the ratio that the coverage area of all the working nodes and the area of the monitoring area is called the effective coverage rate.

$$
C P=\frac{\sum\left(\bigcup_{i \in V}\left(s_{i}\right) \cap\left(s_{i}, s_{j}\right)\right)}{\operatorname{area}(S)}
$$

Definition 2( $k$-coverage): In the monitoring region, any target node is covered by at least $\mathrm{K}$ sensor nodes, which is called the $k$-coverage of the target node.

Definition 3(Covering set): A set consisting of a covering node is called a covering set.

Theorem 1: The sensor nodes are randomly deployed in the monitoring area according with the Poisson distribution with node density $\lambda$ as parameter, $N(s)$ as random variables; $s$ is the network coverage area of any sensor node, and $S$ is the area of the whole monitoring area.

Prove: The probability of any sensor nodes in the monitoring area is $p, p=|s| / S$. By the binomial theorem, when the $k$ numbers of sensor nodes are in the monitoring area, the joint probability is:

$$
p(N(s)=k)=C_{N}^{k} p^{k}(1-p)^{N-k}
$$

For the entire monitoring area, sensor node density is:

$$
\lambda=N / S
$$

Bring $\lambda$ and $\mathrm{p}$ into formula (2), according to calculations:

$$
p(N(s)=k)=\left(1-\frac{\lambda|s|}{N}\right)^{N} \frac{(\lambda|s|)^{k}}{k !} \frac{N !}{(N-k) !(N-\lambda|s|)^{k}}
$$

When the number of sensor nodes in the monitoring area increase indefinitely, namely: $N \rightarrow \infty$,

$$
p(N(s)=k)=\frac{e^{-\lambda \mid s}(\lambda|s|)^{k}}{k !}
$$

End. 
By Theorem 1, we can know that when a large number of sensor nodes are deployed in the monitoring area, and ensure the independent and uniform distribution of each node, the sensor nodes are randomly deployed in the monitoring area according with the Poisson distribution with node density as parameter, $N(s)$ as random variables.

The number of redundant nodes of sensor nodes directly affects the quality of coverage in the monitoring area [22-23]. The coverage of the monitoring area with the minimum node is another key problem discussed in this paper. When the moving target is covered $N$ times by the sensor nodes in the monitored area, the expected value of the $N+1$ coverage is decided by the merits of the successor coverage, therefore, this paper introduces the theorem 2 .

Theorem 2: When the effective coverage is completed, the minimum number of sensor nodes is: $N=S \ln (1-p) / \pi r^{2}$

Prove: The coverage rate of any node in the monitoring area is: $p=\pi r^{2} / S$, The probability of not being covered is: $q=1-\pi r^{2} / S$. According to theorem 1, we can see that in the whole monitoring area, the joint probability which is not covered by any node is:

$$
p_{u}(N(S)=k)=\frac{e^{-\lambda|S|}(\lambda|S|)^{k}}{k !} \times\left(1-\frac{\pi r^{2}}{S}\right)^{k}
$$

According to calculations:

$$
p_{u}=e^{-\lambda \pi r^{2}}
$$

In the monitoring area, the probability that any location is covered is:

$$
p=1-e^{-\lambda \pi r^{2}}
$$

The node density is substituted into the formula (8), both sides have to take the logarithm:

$$
N \geq \operatorname{Sln}(1-p) / \pi r^{2}
$$

Therefore, when the effective coverage is completed, the minimum number of sensor nodes is: $N=S \ln (1-p) / \pi r^{2}$

End.

\section{System Evaluations}

In order to reflect the simulation results, the simulation parameters are described as follows, as shown in Table 1:

Table 1. Description of Simulation Parameters

\begin{tabular}{cccc}
\hline parameter & value & Parameter & value \\
\hline area I & $100 * 100$ & $R$ & $20 \mathrm{~m}$ \\
area II & $200 * 200$ & $E_{R \text {-elec }}$ & $30 \mathrm{~J} / \mathrm{b}$ \\
areaIII & $300 * 300$ & $E_{T-\text { elec }}$ & $30 \mathrm{~J} / \mathrm{b}$ \\
$r$ & $10 \mathrm{~m}$ & $\varepsilon_{f s}$ & $10(\mathrm{~J} / \mathrm{b}) / \mathrm{m} 2$ \\
$\varepsilon_{\text {amp }}$ & $100(\mathrm{~J} / \mathrm{b}) / \mathrm{m}^{2}$ & energy & $2 \mathrm{~J}$ \\
$e_{\min }$ & $0.005 \mathrm{~J}$ & time & $100 \mathrm{~ms}$ \\
\hline
\end{tabular}

Experiment 1: Experimental environment is WIN XP; RAM 2G, Dual-core CPU 1.7 G, with the node density $\lambda$ as the parameter, the optimization of the number of nodes is completed in different monitoring areas, as shown in Figure 1 to figure 6: 


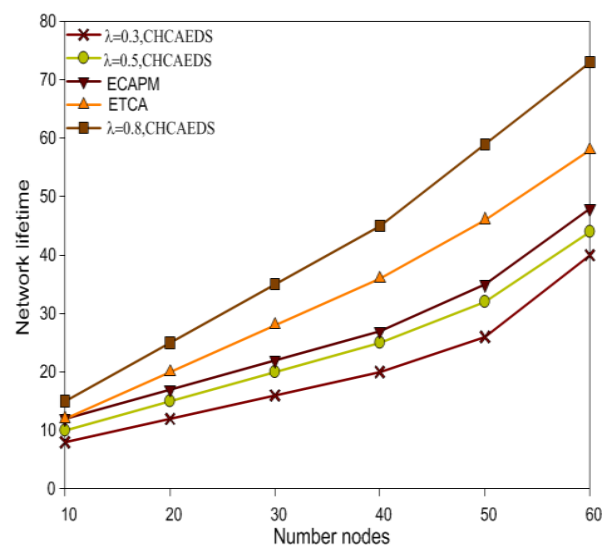

Figure 1. $100^{\star} 100$ Comparison of Sensor Nodes and Network Lifetime

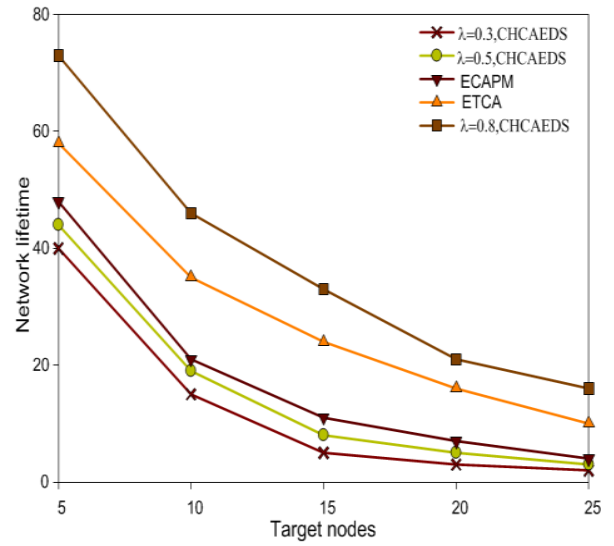

Figure 2. $100 * 100$ Comparison of Target Node and Network Survivability

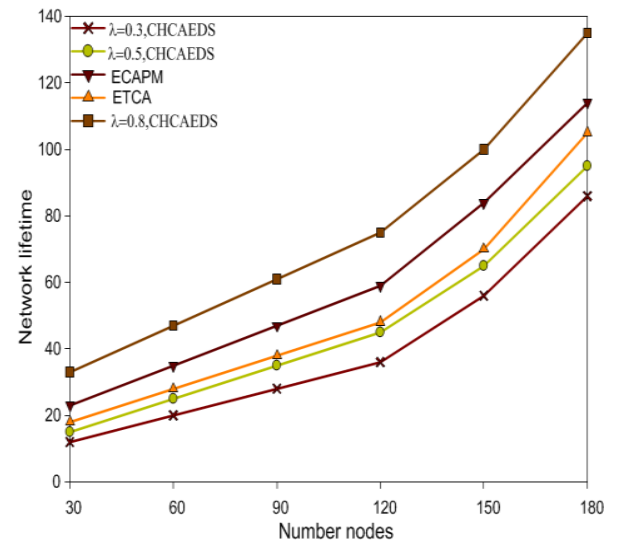

Figure 3. $200 * 200$ Comparison of Sensor Nodes and Network Lifetime 


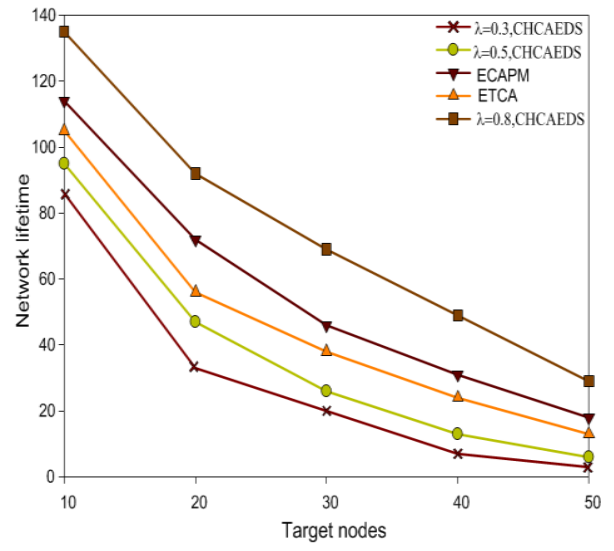

Figure 4. $200 * 200$ Comparison of Target Node and Network Survivability

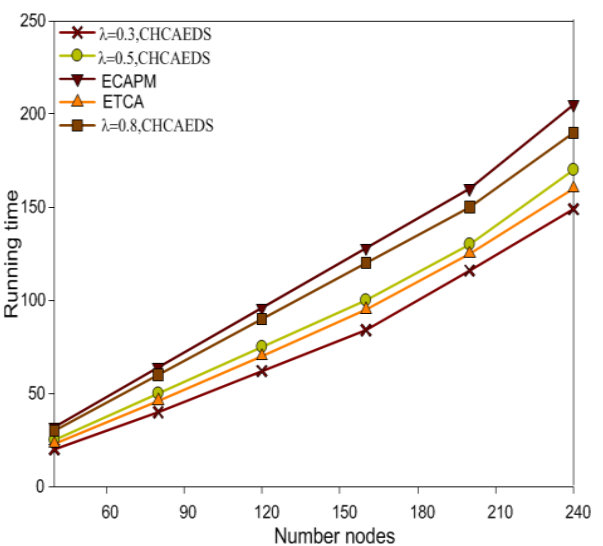

Figure 5. 300*300 Comparison of Sensor Nodes and Algorithm Running Time

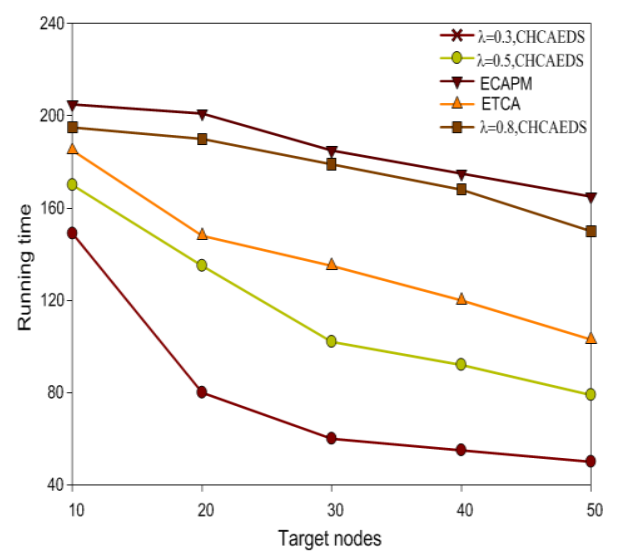

Figure 6. $300 * 300$ Comparison of Target Node and Algorithm Running Time

In this paper, from figure 1 to 6 , the CHCAEDS algorithm is compared with energyefficient target coverage algorithm (ETCA) [17] and enhanced coverage algorithm Based on probability model (ECAPM) [21] in the network lifetime and algorithm running time. Figure 1 and figure 3 respectively give the comparison of the sensor nodes and the network lifetime in the $100 * 100$ and $200 * 200$. As can be seen from the graph, with the increase of the number of sensor nodes, the network lifetime is also steadily improved. When the sensor nodes are 50 and 150, the average value of the network lifetime of CHCAEDS algorithm is higher than that of the other two algorithms $12.59 \%$. Figure 2 
and Figure 4 give the comparison of the target node and the network lifetime. As can be seen from the graph, when the target node is increased, the number of sensor nodes which need to cover the target node is increased, and the energy consumption of the network is increased, so the whole network lifetime is decreased. Compared with the other two algorithms, the descent speed of CHCAEDS algorithm is relatively slow, at the same time, the network lifetime is prolonged, and the average value is $10.82 \%$. Figure 6 and Figure 7 give the comparison of the algorithm running time of the sensor nodes and the target nodes in the $300 * 300$. As can be seen from the graph, when the number of sensor nodes increases, the running time of the algorithm will also increase, but the running time of the algorithm CHCAEDS is smaller than the algorithm ECAPM. The main reason is that the ECAPM is dependent on the increase of the number of sensor nodes, so as to improve the coverage of the monitoring area and ignore the existence of redundant nodes. However, the CHCAEDS use the conversion between the nodes to achieve coverage of the balance. Therefore, the running time of the algorithm CHCAEDS is less than the other two algorithms, and vice versa.

Experiment 2: The simulation experiment of the state of the sensor node is carried out with the $300 * 300$ monitoring area.

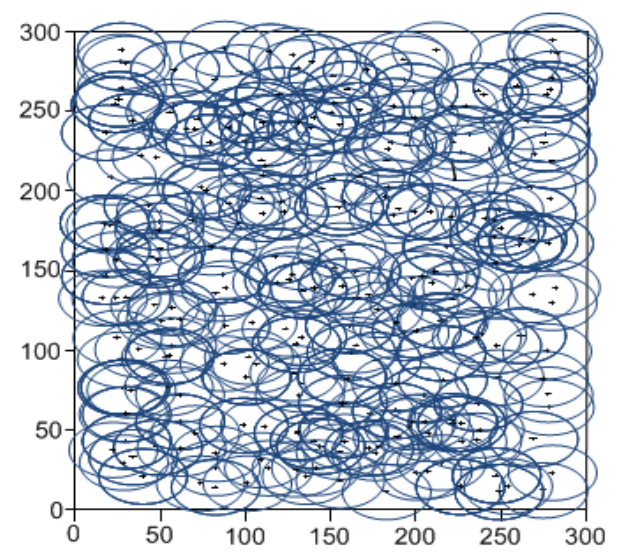

Figure 7. When $\lambda=0.3$, Initial Deployment Diagram

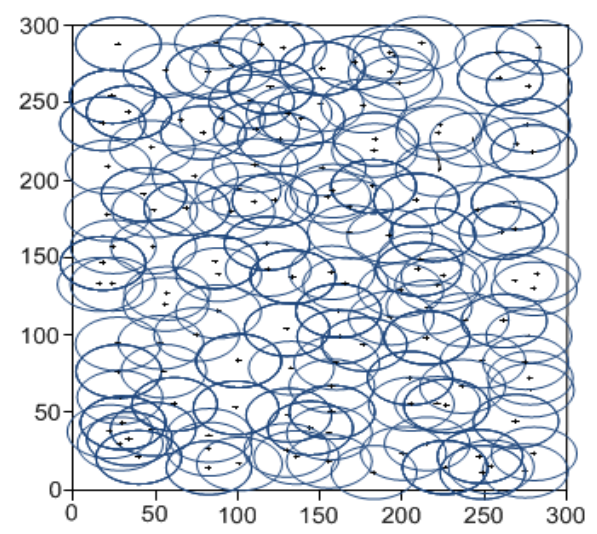

Figure 8. When $\lambda=0.3$, Deployment Diagram of CHCAEDS Algorithm 


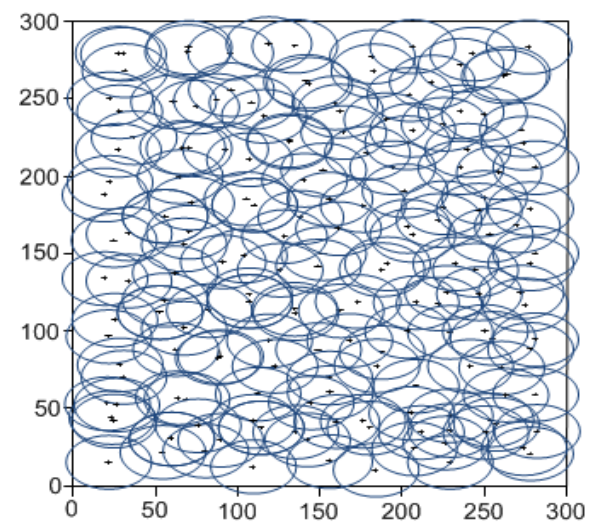

Figure 9. When $\lambda=0.8$, Initial Deployment Diagram

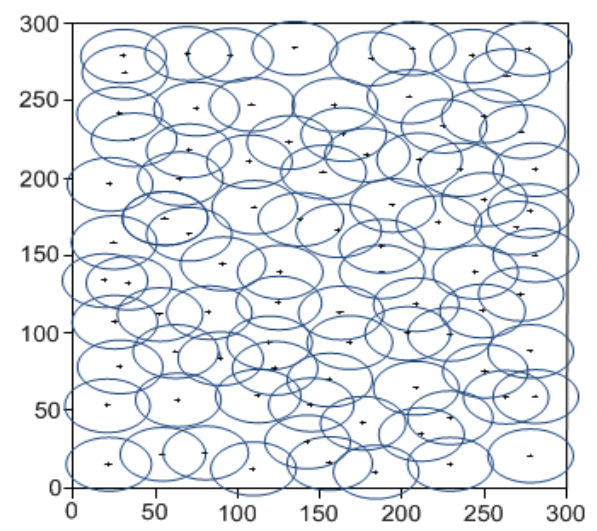

\section{Figure 10. When $\lambda=0.8$, Deployment Diagram of CHCAEDS Algorithm}

Figure 7 to figure 10 shows the comparison of the sensor nodes in the initial time and the optimization of the CHCAEDS algorithm. As can be seen from the graph, when $\lambda=0.3$ and $\lambda=0.8$, there are a lot of redundant nodes in the monitoring area. Through the optimization of CHCAEDS algorithm, the generation of redundant nodes is suppressed. The energy of the whole network is saved, and the network lifetime is prolonged.

\section{Conclusions}

On the basis of analyzing the coverage problem of wireless sensor networks, this paper proposes a Coverage Holes Compensation Algorithm Based on Event-Driven Strategy. Firstly, the distribution pattern of the sensor nodes deployed in the monitoring area is given by using probability knowledge; Secondly, to solve the problem of the function relationship between the expected value and the minimum number of sensor nodes, a method for solving the minimum number of sensor nodes in the coverage area is presented; Finally, the simulation results show that the CHCMEDS algorithm can improve the network lifetime and the running time. The validity and stability of the algorithm is proved.

In the future, the focus of the work is mainly focused on the nonlinear cover of the boundary effect and the effective coverage of the adjustable parameters.

\section{Acknowledgments}

Projects (61503174, U1304603) supported by the National Natural Science Foundation of China; Project (14B520099, 16A520063) supported by Henan Province Education 
Department Natural Science Foundation; Project (142102210471) supported by Natural Science and Technology Research of Foundation Project of Henan Province Department of Science.

\section{References}

[1] Y. Xiao, H. Chen, K. Wu, B. Sun, Y. Zhang, X. Y. Sun and C. Liu, "Coverage and Detection of a Randomized Scheduling Algorithm in Wireless Sensor Networks", IEEE Transactions on Computers, vol. 59, no. 4, (2010), pp. 507-521.

[2] Y. Changlin and C. Kwanwu, "Novel algorithm for complete targets coverage in energy harvesting wireless sensor networks", IEEE Communications Letters. vol. 18, no. 1, (2014), pp. 118-121.

[3] J. S. Su, W. Z. Guo, C. L. Yu and G. L. Chen, "Fault-tolerance clustering algorithm with load-balance aware in wireless sensor network", Chinese Journal of Computers, vol. 37, no. 2, (2014), pp. 445-456.

[4] X. F. Xing, G. J. Wang, J. Wu and J. Li, "Square region-based coverage and connectivity probability model in wireless sensor networks", CollaborateCom 2009. IEEE 5th International Conference Collaborative Computing Networking, Applications and Worksharing, USA Washington: IEEE Proceedings, (2009), pp. 1-8.

[5] Y. C. Tseng, P. Y. Chen and W. T. Chen, "k-Angle Object Coverage Problem in a Wireless Sensor Networks", IEEE Sensor Journal, vol. 12, no. 12, (2012), pp. 3408-3416.

[6] R. S. Mustapha, M. Abdelhamid, O. Latifaa and A. Amar, "An Evidence-Based Sensor Coverage Model”, IEEE Communications Latters, vol. 16, no. 9, (2012), pp. 1462-1465.

[7] J. Chen, L. Zhang and Y. H. Kuo, "Coverage-Enhancing Algorithm Based on Overlap-Sense Ratio in Wireless Sensor Multimedia Sensor Networks”, IEEE Sensor Journal, vol. 13, no. 6, (2013), pp. 20772013.

[8] S. B. He, X. W. Gong and J. S. Zhang, "Curve-Based Deployment for Barrier Coverage in Wireless Sensor Networks", IEEE Transaction Communications, vol. 13, no. 2, (2014), pp. 724-735.

[9] C. F. Cheng and K. T. Tsai, "Distributed Barrier Coverage in Wireless Visual Sensor Networks WithQoM”, IEEE Sensors Journal, vol. 12, no. 6, (2012), pp. 1726-1735.

[10] A. Hossain, S. Chakrabarti and P. K. Biswas, "Impact of Sensor Model on Wireless Sensor Network Coverage", IET Wireless Sensor Systems. vol. 2, no. 3, (2012), pp. 272-281.

[11] R. Tahiry and S. R. David, "Connectivity preservation and coverage schemes for wireless sensor networks", IEEE Trans. on Automatic Control, vol. 56, no. 10, (2011), pp. 2418-2428.

[12] H. M. Ammari and S. K. Das, "Centralized and clustered k-coverage protocols for wireless sensor networks", IEEE Trans. on Computers, vol. 61, no. 1, (2012), pp. 118-132.

[13] J. Z. Du, K. Wang and D. Guo, "Maximizing the lifetime of k-discrete barrier coverage using mobile sensors", IEEE Sensors Journal, vol. 13, no. 12, (2013), pp. 4690-4701.

[14] S. Mini, S. Udgata, S. Sabat, "Sensor Deployment and Scheduling for Target Coverage Problem in Wireless Sensor Networks", IEEE Sensors Journal, vol. 14, no. 3, (2014), pp. 636-644.

[15] Z. Y. Sun, W. G. Wu, H. Z. Wang, H. Chen and W. Wei, "An optimized strategy coverage control algorithm for WSN", International Journal of Distributed Sensor Network, vol. 14, no. 12, (2014), pp. 112 .

[16] Z. Y. Sun, W. G. Wu, H. Z. Wang, H. Chen and X. F. Xing, "A novel coverage algorithm based on event-probability-driven mechanism in wireless sensor network", EURASIP Journal on Wireless Communications and Networking, vol. 20, no. 2, (2014), pp. 1-17.

[17] X. F. Xing, G. J. Wang and J. Li, "Polytype target coverage scheme for heterogeneous wireless sensor network using linear programming", Wireless Communications and Mobile Computing, vol. 14, no. 8, (2014), pp. 1397-1408.

[18] M. Cardei and D. Z. Du, "Improving wireless sensor network lifetime through power aware organization", Wireless Networks, vol. 11, no. 3, (2005), pp. 333-340.

[19] S. H. Yang, F. Dai, M. Cardei, J. Wu and F. Patterson, "On connected multiple point coverage in wireless sensor networks", International Journal of Wireless Information Networks, vol. 13, no. 4, (2006), pp. 289-301.

[20] H. Liu, P. Wan, C. W. Yi, X. H. Jia, S. Makki and P. Niki, "Maximal lifetime scheduling in sensor surveillance networks", INFOCOM 2005. 24th Annual Joint Conference of the IEEE Computer and Communications Societies. USA Miami: IEEE Proceedings, (2005), pp. 2482-2491.

[21] Z. Y. Sun, H. Z. Wang, W. G. Wu and X. F. Xing, "ECAPM: An Enhanced Coverage Algorithm in Wireless Sensor Networks Based on Probability Model", International Journal of Distributed Sensor Networks, vol. 15, no. 3, (2015), pp. 1-11.

[22] M. Hanid, M. Kaveh, G. A. Amir, S. P. Kanran and M. Vladimir, "Distributed deployment algorithms for improved coverage in a network of wireless mobile sensors", IEEE Transactions on Industrial Informatics, vol. 10, no. 1, (2014), pp. 163-174.

[23] T. Samil, U. Numan and K. Okyay, "On Deployment of Wireless Sensor on 3D Terrains to Maximize Sensing Coverage by Utilizing Cat Swarm Optimization with Wavelet Transform", IEEE Transactions on Systems, Man and Cybernetics: Systems, vol. 44, no. 1, (2014), pp. 111-120. 


\section{Authors}
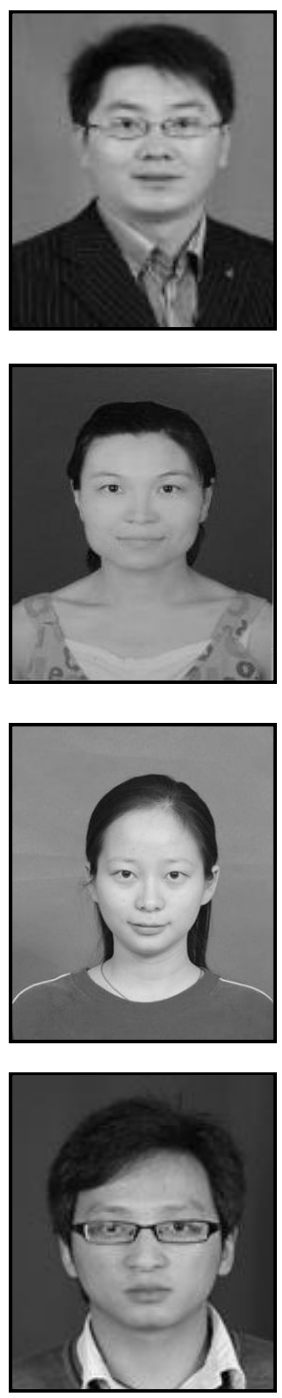

Zeyu Sun, he was born in 1977 in Changchun city, Jilin province, in 2009 graduated from Lanzhou University, Master of Science; Xi'an Jiaotong University study for $\mathrm{PhD}$ at present. $\mathrm{He}$ is associate professor in School of Computer and Information Engineering, Luoyang Institute of Science and Technology, and is also a member of China Computer Society. The main research interest is in wireless sensor networks, parallel computing and Internet of things.

Yali Yun, she was born in 1975 in Luoyang city Henan province, in 2004 graduated from Southeast University, Master of Science; She is a lecturer in College of Computer and Information Engineering, Luoyang Institute of Science and Technology. The main research interest is artificial intelligence, cloud computing.

Yalin Nie, she was born in 1981 in Yiyang city, Hunan province. She was graduated from Xidian University, Ph. D., in 2015. She is a lecturer in School of Computer and Information Engineering, Luoyang Institute of Science and Technology, and is also a member of China Computer Society. Her main research interest is in wireless sensor networks, internet of things and machine learning.

Yuanbo Li, he was born in 1988 in Luoyang city, Henan province, in 2015 graduated from Shaanxi Normal University, Master of Science. The main research interest is in wireless sensor networks, parallel computing and Internet of things. 